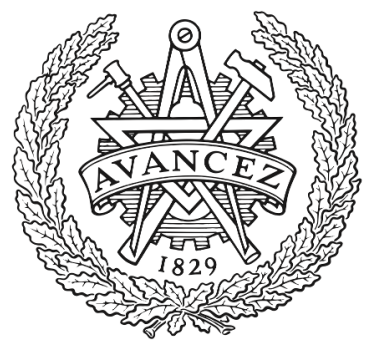

CHALMERS

UNIVERSITY OF TECHNOLOGY

\title{
Subgap current in superconducting tunnel junctions with diffusive electrodes
}

Downloaded from: https://research.chalmers.se, 2023-04-26 12:10 UTC

Citation for the original published paper (version of record):

Bezugly, E., Vasenko, A., Bratus', E. et al (2006). Subgap current in superconducting tunnel junctions with diffusive electrodes. Physical Review B, 73. http://dx.doi.org/10.1103/PhysRevB.73.220506

N.B. When citing this work, cite the original published paper. 


\title{
Subgap current in superconducting tunnel junctions with diffusive electrodes
}

\author{
E. V. Bezuglyi, ${ }^{1,2}$ A. S. Vasenko, ${ }^{2,3}$ E. N. Bratus, ${ }^{1}$ V. S. Shumeiko, ${ }^{2}$ and G. Wendin ${ }^{2}$ \\ ${ }^{1}$ Institute for Low Temperature Physics and Engineering, Kharkov 61103, Ukraine \\ ${ }^{2}$ Chalmers University of Technology, S-41296 Göteborg, Sweden \\ ${ }^{3}$ Department of Physics, Moscow State University, Moscow 119992, Russia
}

(Received 22 February 2006; published 29 June 2006)

\begin{abstract}
We calculate the subgap current in planar superconducting tunnel junctions with thin-film diffusive leads. It is found that the subharmonic gap structure of the tunnel current scales with an effective tunneling transparency which may exceed the junction transparency by up to two orders of magnitude depending on the junction geometry and the ratio between the coherence length and the elastic scattering length. These results provide an alternative explanation of enhanced values of the subgap current in tunneling experiments often ascribed to imperfection of the insulating layer. We also discuss the effect of finite lifetime of quasiparticles as the possible origin of additional enhancement of multiparticle tunnel currents.
\end{abstract}

DOI: 10.1103/PhysRevB.73.220506

Subgap quasiparticle current in superconducting junctions at small applied voltages $e V<2 \Delta$ is the subject of persistent theoretical interest and experimental research. Recently, the problem has attracted new attention, and a number of measurements of the subgap current in high-quality tunnel junctions have been performed, ${ }^{1,2}$ motivated by the problem of decoherence in Josephson-junction-based superconducting qubits. $^{3}$ The subgap current at zero temperature is due to multiparticle tunneling (MPT) processes, ${ }^{4}$ whose intensities strongly depend on the quality of the insulating layer, being enhanced by disorder, localized electronic states, pinholes, etc. ${ }^{5}$ The effect of disorder in the junction electrodes on the subgap current has never been questioned.

According to the MPT theory, ${ }^{4}$ the subgap tunnel current depends on the transparency $D$ of the tunnel barrier: it decreases with decreasing voltage in a steplike fashion with step heights proportional to $(D / 2)^{n}$ at voltages $e V=2 \Delta / n$, $n=1,2, \ldots$ [subharmonic gap structure (SGS)]. Similar results have been obtained for junctions with ballistic electrodes, ${ }^{6}$ and mesoscopic point contacts with diffusive electrodes $^{7}$ on the basis of the theory of multiple Andreev reflections (MARs). ${ }^{5}$

Experimentally, the SGS scaling parameter in atomic size junctions nicely agrees with the theory ${ }^{8}$ however, in macroscopic tunnel junctions it is usually much larger ${ }^{1,2}$ (see also earlier data ${ }^{9}$ ); moreover, there is a smooth residual current at a very low voltage. ${ }^{1}$ Although enhanced SGSs in hightransmission junctions could be explained by assuming randomly distributed resonant levels within the tunnel barrier, ${ }^{10}$ enhanced subgap current in low-transmission junctions with presumably good insulating layers remains an open question.

In this paper we reexamine the problem of the subgap current in macroscopic tunnel junctions, and consider the effects of diffusive electrodes and planar junction geometry common for the experiment (see Fig. 1). Our main result is that the SGS scaling parameter for such junctions significantly exceeds the junction transparency: for the sandwichtype junction with thin-film leads shown in Fig. 1(b), the scaling is determined by the effective transparency defined as

$$
D_{e f f}=\left(3 \xi_{0}^{2} / \ell d\right) D
$$

where $\xi_{0}=\sqrt{\mathcal{D} / 2 \Delta}$ is the diffusive coherence length (we assume $\hbar=k_{B}=1$ ), $\ell$ is the elastic mean free path, $d \ll \xi_{0}$ is the
PACS number(s): 74.45.+c, 74.25.Fy, 74.40.+k, 74.50.+r

thickness of the leads, and $\mathcal{D}$ is the diffusion coefficient. For Al junctions with $\ell \sim d=50 \mathrm{~nm}$ and $\xi_{0}=300 \mathrm{~nm}$, the enhancement factor approaches 100. For the junctions with one-dimensional (1D) geometry of Fig. 1(a), $D_{\text {eff }}$ $=\left(3 \xi_{0} / \ell\right) D .{ }^{11}$ This result also applies to nonhomogeneous tunnel barriers as soon as the size of pinholes (more transparent spots) exceeds the elastic mean free path, otherwise the ballistic scaling ${ }^{6}$ will be valid.

The enhancement effect can be qualitatively understood by considering a short point contact with the reservoirs located very close to the contact, $L \ll \xi_{0}$ [cf. Fig. 1(a)]. In this case, the current can be calculated within the mesoscopic approach, ${ }^{12}$ by integrating over contributions of normal conducting eigenmodes with randomly distributed transparencies. The relevant distribution is known to be spread over the interval $\sim(L / \ell) D \gg D .{ }^{13}$ The most transparent modes dominate the subgap current, giving $D_{e f f} \sim(L / \ell) D$. In our case of junctions with large distance to the reservoirs, the scale of the spatial variation of the Green's function $\xi_{0}$ plays the role of the effective junction length giving qualitatively our result, $D_{\text {eff }} \sim\left(\xi_{0} / \ell\right) D .{ }^{14}$ We note that for the long junctions under consideration the statistics of the eigenmode transparencies is not known, and a quantitative result has to be derived from the quasiclassical theory for diffusive superconductors.

Our analysis is based on the diffusive equations ${ }^{15}$ for the quasiclassical two-time Keldysh-Green functions $\check{G}\left(\boldsymbol{r}, t_{1}, t_{2}\right)$,

$$
[\check{H}, \circ \check{G}]=i \mathcal{D} \nabla \check{J}, \quad \check{G} \circ \check{G}=1, \quad \check{G}=\left(\begin{array}{cc}
\hat{g}^{R} & \hat{G}^{K} \\
0 & \hat{g}^{A}
\end{array}\right) .
$$

Here $\check{H}\left(t_{1}, t_{2}\right)=\left[i \sigma_{z} \partial_{t_{1}}+\Delta \exp \left(i \sigma_{z} \phi\right) i \sigma_{y}\right] \delta\left(t_{1}-t_{2}\right), \phi$ is the superconducting phase, the sign $\circ$ denotes time convolution,

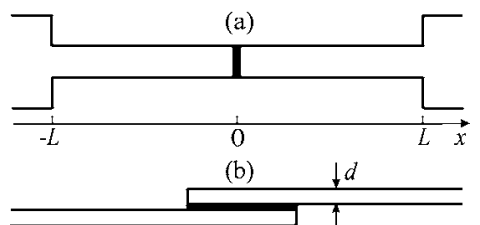

FIG. 1. One-dimensional (a) and planar (b) models of the tunnel junction with diffusive leads; equilibrium reservoirs are far from the contact, $L \gg \xi_{0}$. 
and $\check{J}=\check{G} \circ \nabla \check{G}$. Equation (2) can be decomposed into the Usadel equation for the retarded or advanced Green's functions $\hat{g}^{R, A}$ and the equation for the Keldysh function $\hat{G}^{K}=\hat{g}^{R} \circ \hat{f}-\hat{f}$ $\circ \hat{g}^{A}$, where $\hat{f}=f+\sigma_{z} f_{-}$is the distribution function.

We present detailed calculations for the simpler, 1D geometry of Fig. 1(a). At the left electrode, $x=-L$, the Fourier transformations of the two-time functions $\hat{g}$ and $\hat{f}$ with respect to the variable $t_{1}-t_{2}$ are given by the equilibrium expressions

$$
\begin{aligned}
& \hat{g}(E)=\sigma_{z} u(E)+i \sigma_{y} v(E), \quad \hat{f}(E)=\tanh (E / 2 T), \\
& (u, v)=(E, \Delta) / \xi(E), \quad \xi^{R, A}=\left[(E \pm i 0)^{2}-\Delta^{2}\right]^{1 / 2} .
\end{aligned}
$$

At the right voltage-biased electrode, $x=L$, the function $\check{G}$ is defined through the gauge transformation ${ }^{16}$

$$
\check{G}(L)=\check{\check{G}}(-L) \equiv S\left(t_{1}\right) \check{G}(-L) S^{\dagger}\left(t_{2}\right),
$$

with a unitary operator $S(t)=\exp \left[i \sigma_{z} \phi(t) / 2\right]$, where the phase $\phi$ satisfies the Josephson relation $\phi(t)=2 \mathrm{eVt}$.

The boundary conditions ${ }^{17}$ for the functions $\check{G}$ and $\check{J}$ at the tunnel barrier $(x= \pm 0)$ are given by the relations

$$
\check{J}_{-0}=\check{J}_{+0}=\frac{W}{\xi_{0}}\left[\check{G}_{-0}, \circ \check{G}_{+0}\right], \quad W=\frac{R_{0}}{2 R}=\frac{3 \xi_{0}}{4 \ell} D,
$$

where $R$ is the resistance of the tunnel barrier, $R_{0}=\xi_{0} / g$ is the resistance of a piece of the lead with length $\xi_{0}$, and $g$ is the conductance of the leads per unit length. Assuming a small value of the tunneling parameter $W$, we neglect the charge imbalance function $f_{-}$and the superfluid momentum within the leads, as well as the variation of $\Delta$. In such an approximation, Eq. (5) extends to the whole right lead, $\breve{G}(x)$ $=\bar{G}(-x)$ for $0<x<L$. The problem is therefore reduced to the solution of a static equation for the function $\breve{G}\left(x, t_{1}, t_{2}\right)$ at $-L<x<0$ with the time-dependent boundary condition (6) at $x=-0$. The electric current is related to the Keldysh component $\hat{J}^{K}$ of the matrix current $\breve{J}$ as $I(t)$ $=(\pi g / 4 e) \operatorname{Tr} \sigma_{z} \hat{J}^{K}(x, t, t) .{ }^{15}$ Using Eqs. (5) and (6), it can be expressed as

$$
I(t)=(\pi / 8 e R) \operatorname{Tr} \sigma_{z}[\check{G}, \circ \overline{\bar{G}}]^{K}(t, t) .
$$

In this and following equations, the functions are taken at the boundary $x=-0$. Expanding all functions over harmonics of the Josephson frequency, $A(E, t)=\Sigma_{m} A(E, m) e^{-2 i e V m t}\left[t=\left(t_{1}\right.\right.$ $\left.\left.+t_{2}\right) / 2\right]$, we arrive at the equation for the dc current $I$,

$$
\begin{aligned}
I= & \frac{1}{16 e R} \int_{-\infty}^{\infty} d E \operatorname{Tr} \sum_{m}\left[\hat{h}(E, m) \overline{\hat{G}^{K}}(E,-m)-\overline{\hat{h}}(E, m)\right. \\
& \left.\times \hat{G}^{K}(E,-m)\right], \quad \hat{h}=\sigma_{z} \hat{g}^{R}-\hat{g}^{A} \sigma_{z} .
\end{aligned}
$$

In the tunneling limit $W \ll 1$, the amplitude of the $m$ th harmonic is proportional to $W^{m}$; thus the zero harmonic $m$ $=0$ of the functions $\hat{g}$ and $\hat{G}^{K}$ in Eq. (8) plays the key role, while the high-order harmonics can be neglected. The effect of these harmonics will be discussed later. Within this approximation, the Green's function matrix structure in Eq. (3) holds, and the current Eq. (8) exactly transforms to the form

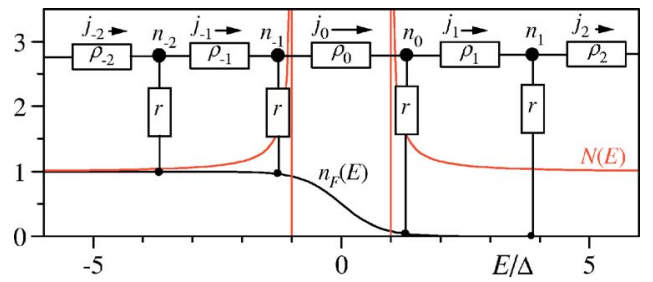

FIG. 2. (Color online) Circuit representation of charge transport in a diffusive tunnel junction, $e V=2.5 \Delta$.

$$
I=\frac{1}{e R} \int_{-\infty}^{\infty} d E N(E) N(E-e V)[n(E-e V)-n(E)] .
$$

Here $N(E)=\operatorname{Re} u^{R}$ is the density of states (DOS) normalized to its value in the normal state, and the distribution function $n=(1 / 2)(1-f)$ approaches the Fermi function $n_{F}$ in equilibrium. Furthermore, we split the integral in Eq. (9) into pieces of length $e V$, denoting $A_{k}(E) \equiv A(E+k e V)$,

$$
\begin{gathered}
I=\frac{1}{e R} \int_{0}^{e V} d E J(E), \quad J(E)=\sum_{k=-\infty}^{\infty} j_{k}(E), \\
j_{k}=\left(n_{k-1}-n_{k}\right) \rho_{k}^{-1}, \quad \rho_{k}^{-1}=N_{k} N_{k-1} .
\end{gathered}
$$

The distribution function $n(E, x=-0)$ satisfies the recurrence relation following from the kinetic equation $\partial_{x}\left(D_{+} \partial_{x} n\right)=0$,

$$
\Theta\left(\left|E_{k}\right|-\Delta\right)\left[n_{F}\left(E_{k}\right)-n_{k}\right]=r\left(j_{k+1}-j_{k}\right),
$$

where $\Theta(x)$ is the Heaviside step function, $r=R_{N} / R \ll 1$, and $R_{N}$ is the normal resistance of the lead. To justify Eq. (12), we note that the diffusion coefficient $D_{+}=(1 / 2)\left(1+|u|^{2}\right.$ $\left.-|v|^{2}\right)$ is approximately constant, $D_{+} \approx 1$, at $|E|>\Delta$, which leads to the linear function $n(E, x)=n_{-0}+(x / L)\left(n_{-0}-n_{F}\right)$. At $|E|<\Delta, D_{+}$turns to zero at $|x| \gg \xi_{0}$, which reflects complete Andreev reflection in the leads and results in zero probability current $D_{+} \partial_{x} n=0$. Then, using the boundary condition at the tunnel barrier following from Eq. (6), $D_{+} \partial_{x} n$ $=\left(2 \mathrm{~W} / \xi_{0}\right) \sum_{k= \pm 1} N N_{k}\left(n_{k}-n\right)$, we arrive at Eq. (12).

A convenient interpretation of Eqs. (11) and (12) in terms of circuit theory ${ }^{18}$ is given by an infinite network in the energy space with the period $e V$, graphically presented in Fig. 2 . The electric current spectral density $J(E)$ consists of partial currents $j_{k}$, which flow through the tunnel "resistors" $\rho_{k}$ connected to adjacent nodes of the network having "potentials" $n_{k}$ and $n_{k-1}$. At $|E|>\Delta$, the nodes are also attached to the distributed "equilibrium source" $n_{F}(E)$ through equal resistors $r$. Below we impose the equilibrium quasiparticle distribution at $|E|>\Delta, n(E)=n_{F}(E)$, neglecting the effect of small resistors $r$.

The currents flowing between the nodes outside the gap are related to the thermal current; at $T=0$, these nodes have equal populations $\left(n_{k}=1\right.$ at $E_{k}<-\Delta, n_{k}=0$ at $\left.E_{k}>\Delta\right)$; thus the corresponding partial currents are zero, and the thermal current vanishes. As a result, only the current $j_{0}$ flowing across the gap through the resistor $\rho_{0}$ survives at $T=0$.

Taking the DOS in the BCS form $N=N_{S} \equiv \operatorname{Re}\left(E / \xi^{R}\right)$, we see that if any node falls into the gap, the adjacent resistances turn to infinity, and the current vanishes. For this reason, the network period must exceed the gap, $e V>2 \Delta$, and the integration in Eq. (10) is confined to the region $\Delta<E<e V-\Delta$. 


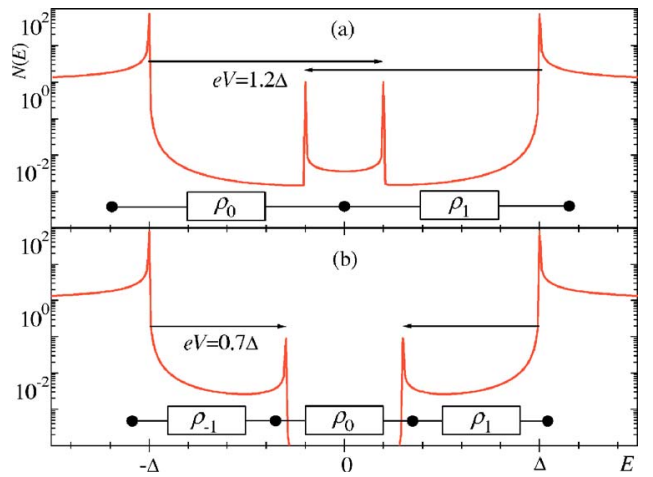

FIG. 3. (Color online) DOS and subgap circuits at the applied voltages $e V=1.2 \Delta$ (a) and $0.7 \Delta$ (b), for the tunneling parameter $W=10^{-3}$.

This recovers the tunneling model result for the singleparticle tunnel current: ${ }^{19}$ the current appears above the threshold, $e V=2 \Delta$, having the threshold value $I_{1}(2 \Delta)$ $=\pi \Delta / 2 e R$.

To evaluate the subgap current, $e V<2 \Delta$, the DOS must be calculated to next order in the parameter $W$, which requires solution of the equations for $\hat{g}$ following from Eqs. (2) and (6). Using the standard parametrization $\hat{g}=\sigma_{z} e^{\sigma_{x} \theta}$, we obtain the equation and the boundary condition for the spectral angle $\theta$,

$$
\begin{gathered}
\sinh \left(\theta-\theta_{S}\right)=i \partial_{z z} \theta \sinh \theta_{S}, \quad z=x / \xi_{0}, \\
\partial_{z} \theta+W \sinh \theta\left(\cosh \theta_{1}+\cosh \theta_{-1}\right)=0 \quad(z=-0) .
\end{gathered}
$$

With exponential accuracy, the solution of Eq. (13) for $z$ $<0$ can be approximated by the formula for a semi-infinite wire,

$$
\tanh \left\{\left[\theta(z)-\theta_{S}\right] / 4\right\}=\tanh \left[\left(\theta_{-0}-\theta_{S}\right) / 4\right] \exp (k z),
$$

where $k^{-1}(E)=\sqrt{i \sinh \theta_{S}}$. Equation (15) describes the decay of perturbations of the spectral functions at distances $\gtrsim \xi_{0}$ from the barrier, where the spectral angle approaches its bulk value $\theta_{S}=\operatorname{arctanh}(\Delta / E)$. The boundary value of $\theta$ is to be found from the equation following from Eqs. (14) and (15),

$2 k \sinh \left[\left(\theta_{S}-\theta\right) / 2\right]=W \sinh \theta\left(\cosh \theta_{1}+\cosh \theta_{-1}\right)$.

A direct expansion of $\theta$ with respect to $W$ in Eq. (16) leads to the following expression for the DOS within the BCS gap,

$N(E)=W\left(1-E^{2} / \Delta^{2}\right)^{-5 / 4}\left[N_{S}(E+e V)+N_{S}(E-e V)\right]$.

The DOS divergencies at $|E|=\Delta, \Delta-e V$ in Eq. (17) are potentially dangerous (cf. Refs. 4), but they can be eliminated by improving the perturbation procedure by solving a set of recurrences in Eq. (16) in the vicinity of these points.

As follows from Eq. (17), the tunneling processes transfer the DOS in the energy space into the BCS gap at the distances $\pm e V$ from the regions $|E|>\Delta$, thus forming an effective spatial potential well of the width $\sim \xi_{0}$ at the tunnel barrier. At $e V>\Delta$ the BCS gap is entirely filled with the quasiparticle states with a small local DOS $\sim W$, as shown in Fig. 3(a). The appearance of localized states enables the quasiparticles to overcome the BCS gap at $e V<2 \Delta$ via two steps involving intermediate Andreev reflection at energies $|E|<\Delta$. The population of the intermediate state cannot be taken to be in equilibrium because the subgap quasiparticles cannot access the equilibrium electrodes. In the circuit terms, the node $k=0$ is disconnected from the equilibrium source, and the subgap current flows through the two large resistances $\rho_{0}, \rho_{1} \sim W^{-1}$ (two-particle current) [see Fig. 3(a)]. The corresponding partial currents are equal, $j_{0}=j_{1}=\left[n_{F}\left(E_{1}\right)\right.$ $\left.-n_{F}\left(E_{-1}\right)\right] /\left(\rho_{0}+\rho_{1}\right)$, and their contribution to $I(V)$ is confined to the energy region $0<E<e V-\Delta$ (a similar contribution at $\Delta<E<e V$ comes from $j_{0}$ and $j_{-1}$ ). Thus the two-particle current appears above the threshold $e V=\Delta$, having the threshold value $I_{2}(\Delta)=\pi W \Delta / e R=2 W I_{1}(2 \Delta)$. At $e V=2 \Delta$, the two-particle current exhibits a sharp peak with the height $I_{2}(2 \Delta) \approx 2.3 W^{2 / 5} \Delta / e R$; at larger voltages, it approaches a constant value giving rise to the excess current $I_{\text {exc }}$ $\approx 6.2 W^{2 / 3} \Delta / e R$.

At $e V<\Delta$, a minigap opens in the DOS around the zero energy [see Fig. 3(b)]; however, since the number of subgap resistors increases up to three (three-particle current), the current across the minigap will persist as long as the network period exceeds the minigap size, $e V>2(\Delta-e V)$, i.e., at $e V$ $>2 \Delta / 3$. The central resistance $\rho_{0}$ is large, $\rho_{0} \sim W^{-2}$, and dominates the net subgap resistance. This leads to a smaller charge current with the threshold value $I_{3}(2 \Delta / 3) \approx 2 W I_{2}(\Delta)$. At $e V<2 \Delta / 3$ the network period becomes smaller than the minigap, and further correction to the DOS is required.

Similar results were found for the planar junction Fig. $1(\mathrm{~b})$, using the equation for the functions $\check{G}_{ \pm 0}$ at the top $(+0)$ and bottom $(-0)$ sides of the tunnel barrier $i\left[\sigma_{z} E\right.$ $\left.+i \sigma_{y} \Delta, \check{G}_{-0}\right]=2 \Delta W\left[\check{G}_{-0}, \check{G}_{+0}\right]$, with the modified tunneling parameter $W=\left(3 \xi_{0}^{2} / 4 \ell d\right) D$. This equation is derived by averaging Eq. (2) over the thickness of overlapping leads and using Eq. (6) (cf. Ref. 20). From this equation we obtain a relation for the spectral angle that does not significantly differ from Eq. (16),

$$
k^{2} \sinh \left(\theta_{S}-\theta\right)=W \sinh \theta\left(\cosh \theta_{1}+\cosh \theta_{-1}\right),
$$

thus giving results which are close to those for the 1D model with the same magnitude of the parameter $W$.

The presented calculation scheme, combining circuit theory arguments with DOS iteration procedures, suggests an appealingly simple explanation for the diffusive SGS: the decreasing applied voltage results in a shrinking period of the network in Fig. 2; hence a stepwise increase of the number of subgap resistors involved; simultaneously, the number of DOS steps, scaled as $W^{n}$, increases, as shown in Fig. 4(a). This results in the current staircase with the height of the steps given by $I_{n} \sim(2 W)^{n-1} I_{1}$, at $2 \Delta / n<e V<2 \Delta /(n-1)$. The quantitative result for the current at arbitrary voltages and temperatures is

$$
\begin{gathered}
I(V)=\int_{0}^{e V} \frac{d E}{e R} \frac{N_{+}+N_{-}}{\rho_{\Delta}}\left(n_{-}-n_{+}\right)+\int_{\Delta}^{\infty} \frac{2 d E}{e R \rho_{1}}\left(n_{F}-n_{F 1}\right), \\
N_{ \pm}=\operatorname{Int}[(\Delta \mp E) / e V]+1, \quad n_{ \pm}(E)=n_{F}\left(E_{ \pm N_{ \pm}}\right) .
\end{gathered}
$$

In this equation, the second term represents the thermal current, the integers $\pm N_{ \pm}$are the indices of the nodes closest to the gap edges outside the gap, $\operatorname{Int}(x)$ denotes integer part of $x$, and the quantity $\rho_{\Delta}(E)=\sum_{k=1-N_{-}}^{N_{+}} \rho_{k}$ has the meaning of net subgap resistance. The subgap distribution function reads 


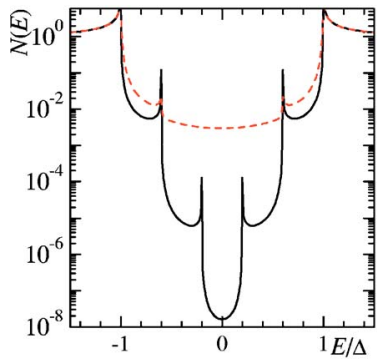

(a)

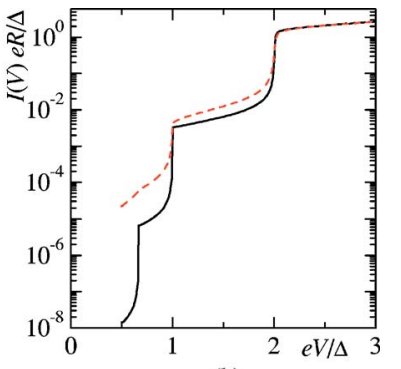

(b)
FIG. 4. (Color online) DOS at $e V=0.4 \Delta$ (a) and $I-V$ characteristics (b) for the tunneling parameter $W=10^{-3}$ and two values of the damping parameter: $\gamma=0$ (solid line) and $\gamma=0.003 \Delta$ (dashed line).

$$
n(E)=n_{+}+\left(n_{-}-n_{+}\right) \rho_{\Delta}^{-1} \sum_{k=1}^{N_{+}} \rho_{k} .
$$

Equations (19) and (20) are the main technical results of the paper. The $I-V$ characteristic (IVC) of the planar tunnel junction calculated from Eqs. (19) and (18) at $T=0$ and shown in Fig. 4(b), was found to be very close to the result for a ballistic point contact ${ }^{6}$ with the effective transparency $D_{e f f}=4 W=\left(3 \xi_{0}^{2} / \ell d\right) D$. This justifies our statement made in the Introduction, and is the main conclusion of this paper.

In low-transmissive junctions, enhanced subgap current at $e V<\Delta$ has been observed (see, e.g., Ref. 1). This anomaly might be due to many-body interaction effects which introduce a finite lifetime (damping) of the quasiparticles. The damping effect can be qualitatively modeled by a small imaginary addition to the energy in the spectral functions, $E \rightarrow E+i \gamma$. This would lead to a small residual DOS within the BCS gap and cut the DOS staircase at the level of the order of $\gamma / \Delta$, see Fig. 4(a). This will result in the smearing of the tunneling SGS and crossover to a linear IVC at low voltages, $I=2.2(\gamma / \Delta)^{2} V / R$, similar to the incoherent MAR regime. ${ }^{18}$ The IVC calculated from Eq. (19) for $\gamma=0.003 \Delta$ and shown in Fig. 4(b) by a dashed line confirms these considerations.

We conclude our analysis with the estimation of the contribution of higher harmonics of the functions $\hat{g}$ and $\hat{G}^{K}$ to the dc current. At $T=0$, the contribution $\delta I$ of the first harmonics $|m|=1$ (the higher harmonics $|m|>1$ are smaller, $\left.\sim W^{m}\right)$ is

$\delta I=\frac{2 W}{e R} \int_{0}^{e V} d E \operatorname{Im} v \operatorname{Im}\left(\frac{v}{p} \cosh ^{2} \frac{\chi}{2}+\frac{v}{q} \sinh ^{2} \frac{\tilde{\chi}}{2}\right)$,

where $\chi=\theta_{1}+\theta_{-1}, \quad \tilde{\chi}=\theta_{1}+\theta_{-1}^{*}, \quad(p, q)^{2}=\left(\xi_{1}^{R}+\xi_{-1}^{R, A}\right) / 2 i \Delta$, and $v=\sinh \theta$. At $e V<\Delta$, the energy $E_{-1}$ appears in the subgap region, where $\theta_{-1}^{*}=\theta_{-1}+\pi i$ and $\xi_{-1}^{A}=\xi_{-1}^{R}$; for this reason, $\delta I$ turns to zero at $e V<\Delta$, similar to $I_{2}$. Numerical calculations show that the contribution of the first harmonics to the IVC does not exceed $30 \%$. From this we conclude that the adopted quasistatic approach gives a rather good approximation to a complete solution.

In our treatment, we have neglected inelastic scattering, which might affect the quasiparticle distribution at subgap energies. Analysis shows that this effect becomes essential under the condition $W \tau_{\epsilon} \Delta \ll 1$, where $\tau_{\epsilon}$ is the relaxation time. However, this does not affect the estimate of the effective scaling factor and only changes the details of the IVC shape.

In conclusion, we have developed a theory of subgap charge transport and subharmonic gap structure in superconducting tunnel junctions with planar geometry and diffusive thin-film electrodes. We found that the role of scaling factor in the subharmonic gap structure is played by the effective tunneling transparency $D_{e f f}=\left(3 \xi_{0}^{2} / \ell d\right) D$, which may greatly exceed the bare transparency $D$ of the junction tunnel barrier.

Support from the Swedish grant agencies SSF OXIDE, VR, and KVA is gratefully acknowledged.
${ }^{1}$ M. A. Gubrud et al., IEEE Trans. Appl. Supercond. 11, 1002 (2001).

${ }^{2}$ K. M. Lang et al., IEEE Trans. Appl. Supercond. 13, 989 (2003); S. Oh et al., Semicond. Sci. Technol. 18, 1396 (2005).

${ }^{3}$ Yu. Makhlin, G. Schön, and A. Shnirman, Rev. Mod. Phys. 73, 357 (2001); G. Wendin and V. S. Shumeiko, in Handbook in Theoretical and Computational Nanoscience, edited by M. Rieth and W. Schommers (American Scientific Publishers, Los Angeles, 2006), Chap. 129 (cond-mat/0508729).

${ }^{4}$ J. R. Schrieffer and J. W. Wilkins, Phys. Rev. Lett. 10, 17 (1963); L. E. Hasselberg, M. T. Levinsen, and M. R. Samuelsen, Phys. Rev. B 9, 3757 (1974).

${ }^{5}$ T. M. Klapwijk, G. E. Blonder, and M. Tinkham, Physica B \& C 109-110, 1657 (1982)

${ }^{6}$ E. N. Bratus', V. S. Shumeiko, and G. Wendin, Phys. Rev. Lett. 74, 2110 (1995); J. C. Cuevas, A. Martin-Rodero, and A. L. Yeyati, Phys. Rev. B 54, 7366 (1996).

${ }^{7}$ E. Scheer, W. Belzig, Y. Naveh, M. H. Devoret, D. Esteve, and C. Urbina, Phys. Rev. Lett. 86, 284 (2001).

${ }^{8}$ B. Ludoph, N. Van der Post, E. N. Bratus', E. V. Bezuglyi, V. S. Shumeiko, and G. Wendin, Phys. Rev. B 61, 8561 (2000).

${ }^{9}$ R. Cristiano, L. Frunzio, R. Monaco, C. Nappi, and S. Pagano, Phys. Rev. B 49, 429 (1994).
${ }^{10}$ Y. Naveh, V. Patel, D. V. Averin, K. K. Likharev, and J. E. Lukens, Phys. Rev. Lett. 85, 5404 (2000).

${ }^{11}$ A similar scaling factor appears in the theory of high-order corrections to the Josephson current: M. Yu. Kupriyanov, JETP Lett. 56, 399 (1992); E. V. Bezuglyi, E. N. Bratus', and V. P. Galaiko, Low Temp. Phys. 25, 167 (1999); A. V. Galaktionov and Chang-Mo Ryu, J. Phys.: Condens. Matter 12, 1351 (2000).

${ }^{12}$ A. Bardas and D. V. Averin, Phys. Rev. B 56, R8518 (1997).

${ }^{13}$ C. W. J. Beenakker, B. Rejaei, and J. A. Melsen, Phys. Rev. Lett. 72, 2470 (1994)

${ }^{14}$ Additional factor $\xi_{0} / d$ in Eq. (1) reflects the "anti-point-contact" geometry of the junctions with overlapping leads and the junction cross section exceeding the one of the leads (see Ref. 20).

${ }^{15}$ A. I. Larkin and Yu. N. Ovchinnikov, Sov. Phys. JETP 41, 960 (1975); 46, 155 (1977).

${ }^{16}$ S. N. Artemenko, A. F. Volkov, and A. V. Zaitsev, Sov. Phys. JETP 49, 924 (1979).

${ }^{17}$ M. Yu. Kupriyanov and V. F. Lukichev, Sov. Phys. JETP 67, 1163 (1988).

${ }^{18}$ E. V. Bezuglyi, E. N. Bratus, V. S. Shumeiko, G. Wendin, and H. Takayanagi, Phys. Rev. B 62, 14439 (2000).

${ }^{19}$ N. R. Werthamer, Phys. Rev. 147, 255 (1966).

${ }^{20}$ A. F. Volkov, Physica B 203, 267 (1994). 